\title{
Correction to: Obtaining Soft Matter Models of Proteins and their Phase Behavior
}

\author{
Irem Altan and Patrick Charbonneau
}

Correction to:

Chapter 15 in: Jennifer J. McManus (ed.), Protein Self-Assembly: Methods and Protocols, Methods in Molecular Biology, vol. 2039, https://doi.org/10.1007/978-1-4939-9678-0_15

The acknowledgement section text has been updated in the chapter.

"We thank Diana Fusco for guiding discussions. The authors acknowledge support from National Science Foundation Grant no. NSF DMR-1749374. This work used the Extreme Science and Engineering Discovery Environment (XSEDE), which is supported by National Science Foundation grant number ACI-1548562, as well as the Duke Compute Cluster.”

The updated online version of this chapter can be found at https://doi.org/10.1007/978-1-4939-9678-0_15 